\title{
A pilot study of the impact of an educational intervention aimed at improving medical record documentation
}

\begin{abstract}
${ }^{1} \mathrm{M}$ Farzandipour, ${ }^{2} \mathrm{Z}$ Meidani, ${ }^{2} \mathrm{~F}$ Rangraz Jeddi, ${ }^{3} \mathrm{H}$ Gilasi, ${ }^{4} \mathrm{~L}$ Shokrizadeh Arani, ${ }^{5} \mathrm{E}$ Fakharian, ${ }^{6} \mathrm{~B}$ Saddik ${ }^{1}$ Associate Professor, Department of Health Information Management/Technology, School of Allied Health Professions, Kashan University of Medical Sciences, Iran; 'Assistant Professor, Department of Health Information Management/Technology, School of Allied Health Professions, Kashan University of Medical Sciences, Iran; ${ }^{3}$ PhD Candidate, Department of Epidemiology, School of Health, Sahid Beheshti University of Medical Sciences and Deputy of Research Kashan University of Medical Sciences, Iran; ${ }^{4}$ PhD Candidate in Health Information Management, School of Allied Health Professions, International Branch, Shahid Beheshti University, Tehran, Iran; ${ }^{5}$ Associate Professor, Department of Surgery, School of Medicine, Kashan University of Medical Science, Kashan, Iran; ${ }^{6}$ Assistant Professor, Department of Health Informatics, College of Public Health and Health Informatics, King Saud bin Abdulaziz University for Health Sciences, Saudi Arabia
\end{abstract}

\section{ABSTRACT}

Introduction: Studies have shown the importance of medical staff education in improving chart documentation and accuracy of medical coding. This study aimed to examine the effect of an educational intervention on recording medical diagnoses among a sample of medical residents based at Kashan University of Medical Sciences. Methods: This pilot study was conducted in 2010 and involved 19 residents in different specialties (internal medicine, obstetrics and gynecology, and surgery). Guidelines for recording diagnostic information related to surgery, obstetrics and internal medicine were taught at a five-hour lecture. Five medical records from each resident from before and after the educational intervention were assessed using a checklist based on relevant diagnostic information related to each discipline. Data were analysed using a paired $t$-test and Wilcoxson signed rank test.

Results: There was no improvement in the quality and accuracy of the recording of obstetric diagnoses (type, place, outcome and complications of delivery) after the training. There was also no effect on the documentation of underlying causes and clinical manifestations of disease by internal medicine and surgery residents $(p=0.285$ and $\mathrm{p}=0.584$, respectively).

Conclusion: The single education session did not improve recording of diagnoses among residents. The gathering and recording of complete, accurate and high quality medical records requires interaction between the hospital management, health information management professionals and healthcare providers. It is therefore essential to develop a more sophisticated portfolio of strategies that involves these key stakeholders.

KEYWORDS Diagnosis writing, medical record, medical records documentation, education, residency training, performance change

DECLARATION OF INTERESTS No conflicts of interest declared

\author{
Correspondence to $\mathbf{Z}$ Meidani, \\ Department of Health \\ Information Management/ \\ Technology, Kashan University of \\ Medical Sciences, Kashan, Iran
}

tel. 00983615558883

e-mail meydani-za@kaums.ac.ir

\section{INTRODUCTION}

Following an episode of hospital care the summary discharge record is an essential documentation of diagnosis and treatment and reflects the overall outcome of the healthcare process. According to the guidelines from the International Classification of Diseases (ICD), diagnostic information should be organised systematically utilising standardised recording methods.' The International Statistical Classification of Diseases and Related Health Problems (ICD-I0, 20I0) allows an international comparison of the collection, classification, processing, and presentation of mortality data collected in different countries or across countries. ${ }^{2-3}$ Classified and coded medical episodes of care can be used for reimbursement assessment, healthcare policy making, ${ }^{4}$ monitoring of the incidence and prevalence of disease, ${ }^{5}$ quality management, ${ }^{6}$ clinical care, education and research. ${ }^{7}$ Researchers often use diagnostic codes from the ICD-9 version to estimate incidence or prevalence of certain health conditions. ${ }^{5}$ Incomplete and/or inadequate use of ICD-9 codes for clinical diagnoses on hospital discharge could lead to an over- or underestimation of disease prevalence. ${ }^{5.8}$ Unreliable or inaccurate clinical coding has major implications for accurate reimbursement. The development of prospective payment systems based on a classification framework which is closely linked with the ICD makes the importance of clinical coding imperative for some hospitals, ${ }^{79-10}$ where hospital revenue will depend to a large extent on the validity of clinical coding and diagnostic information." 
Diagnostic coding continues to be variable., ${ }^{4,12}$ Factors such as clarity of documentation, incomplete information in medical records and lack of attention to detail can lead to unreliable and inaccurate coding. ${ }^{13,14}$ Previous studies have highlighted that inadequacies in morbidity and mortality reports are a direct reflection of shortcomings in the way some conditions are recorded. ${ }^{6,15}$

Geller found that clinicians were directly responsible for the majority of miscoding ( $82.1 \%$ of all errors). In most cases, the nature of diagnostic error was due to a lack of specificity in charting. ${ }^{.}$Improvement in the documentation of diagnosis by the clinicians may therefore increase the coders' ability to collect this information from the medical chart. ${ }^{5.16}$ Findings from other studies support the importance of medical staff in improving chart documentation and in the accuracy of subsequent coding and disease classification., ${ }^{5,17-19}$ There is little previous research addressing the quality of medical record documentation in support of morbidity classification using ICD requirements. Improving coding accuracy through better documentation will provide better quality diagnostic information for clinical, epidemiological, reimbursement and quality purposes; national mortality and morbidity statistics for WHO member states will be more accurate and national health profiles will be better defined.

Our small study was designed to test whether a simple education intervention could improve the recording of health information by residents.

\section{METHODS}

We used an open observational design in a small sample of unselected staff. All protocols were reviewed and approved by the Ethical Review Board of Kashan University of Medical Sciences.

\section{Study setting and population}

The study took place in an academic hospital (the Kashan University of Medical Sciences, located in Esfehan, Iran). Nineteen first-year resident physicians were involved in the research; this included all of the residents who were enrolled at Kashan University for internal medicine, surgical and obstetrics subspecialty residency training.

\section{Study protocol}

The study consisted of three parts: a retrospective chart review, an educational session and a subsequent analysis of physician behaviour following the intervention, one month later.

\section{Chart review}

A team of four reviewers, including physicians and a health information management specialist was established. Five medical records from each resident were assessed by two reviewers from this team; they were blinded to the identity of the recording resident or the planned educational session. Reviewers completed a checklist of items of diagnostic information that should be included in a complete medical chart. It was based on a generic record review form, adapted for this purpose. ${ }^{20}$ Recorded items were scored with one point while missed items scored zero. The checklist was completed again following the educational intervention.

A baseline audit of these assessments confirmed a range of charting deficiencies and the lack of information leading to errors in diagnosis. The main shortcomings which compromised medical coding and caused difficulties in assigning a more specific ICD code in obstetrics were: I) type of delivery including spontaneous, delivery by forceps (e.g. low, mid-cavity) and vacuum, caesarean section and multiple delivery; 2) place of delivery including in hospital, or home; 3 ) outcome of delivery including single, twin, and multiple along with stillbirth and live born;4) delivery complications including laceration, fetus with cord around neck, etc; 5) the type of induction (medical/surgical); 6) stages of prolonged labour; 7) reasons for obstructed labour; 8) types of abortion e.g. medical, spontaneous, and illegal, complete and incomplete abortion; 9) complications following abortion and ectopic and molar pregnancy; 10) and onset of labour, critical for distinguishing conditions which can arise both during pregnancy and during labour and delivery.

In internal medicine the recorded diagnoses rarely considered the aetiology of disease, focusing more often on aspects of the investigation. Localised infections caused by micro-organisms were rarely recorded for example. Where an episode of care involved a range of conditions it was often impossible to distinguish the main condition and other conditions. Primary and secondary spread were not clearly recorded in cancer cases and in the absence of pathology reports, it was often difficult to determine the exact histology and extent of the tumour spread.

\section{Educational intervention}

\section{Design}

The educational session was designed based on the baseline data collection and the problems that were identified. Students attended a five-hour lecture on documentation and medical coding. We initially posed a number of questions aimed at encouraging residents to consider the role and need for accurate documentation: what happens to the patient record when the episode of care finishes; the value of the medical record for research; the importance of an accurate diagnosis when looking within thousands of records; and the role of morbidity data in epidemiology and clinical research was 
emphasised. We wanted to shift the focus from treatment to prevention of diseases with a particular mention of the uses of aggregate data in determining the health of a population. These data are extracted from patientspecific medical records that they are responsible for compiling. A brief review of the post-discharge medical chart lifecycle through an overview of medical record department functions (especially coding) was presented. We also explained the arrangement of recorded data by ICD- 10 code. Residents examined three volumes of the ICD and received a small amount of training on how it is applied in medical coding. Basic cases were presented and residents were asked to find the relevant codes in the ICD. They were largely successful in assigning the correct code and enjoyed this part of the session. The residents gained an understanding of how failing to record detailed diagnostic information impacts negatively on medical coding, medical research and therefore public health monitoring.

\section{The learning objectives}

The learning objectives of the session were limited to the problem areas identified: describing the main diagnosis and ancillary conditions; clarifying the extent of neoplasm (morphology, histology etc.) and to specifying the type, place, outcome and complications of delivery for obstetric conditions. There was no intention to teach medical management or theory, simply to reinforce the importance of recording what they had defined.

\section{Exam}

After the educational session the residents completed a brief exam where copies of medical records and clinical information were provided and they were asked to correct recording errors. Residents within the same subspecialty undertook teamwork exercises aimed at embedding best practice; reviewing real medical records (with the identity of the patient and treating physician kept anonymous). Patient history and clinical information from some cases were presented and a diagnosis statement was recorded by residents.

\section{Prospective analysis}

The third phase of the study assessed the impact of the training session on physician behaviour. The same individual chart review process as the baseline phase was conducted one month after the educational session. The same checklist was used. Data were analysed using a paired t-test for non-normal data; the Wilcoxson nonparametric test was also used.

\section{RESULTS}

The rank scores for diagnostic accuracy are listed in Table I. These observations indicated that the postintervention documentation of main diagnoses and other ancillary conditions was not statistically significant compared with pre-intervention diagnoses. There were no statistically significant changes in the recording of the type, place, outcome or complications of delivery. Recording diagnostic information to support classification of morbidity conditions based on ICD requirements had not improved after intervention $(\mathrm{p}=0.886)$.

\section{DISCUSSION}

Recording accurate and complete diagnostic information is a clinically important task in any healthcare setting. Little previous research has addressed the quality of documentation. Previous healthcare research has suggested chart-based educational training but the effectiveness of this is unclear. We found that one brief educational session was not an effective strategy to change a physician's behaviour and improve the recording of the diagnoses. This contrasts with other research which has proven educational intervention to be an effective means of improving providers' behaviour regarding medical record documentation. ${ }^{21-23}$

Our study results are consistent with those of the Socolar et al. study that showed that providing specific educational materials to physicians did not ensure improvement in medical record documentation. ${ }^{24}$ Greco et al. also found that there is no single best way to improve physicians' practices and that a combination of methods may have greater impact on behaviour. ${ }^{25}$ Tinsley showed that documentation by residents may be improved following education if it was reinforced with other means such as the support of faculty, departmental leadership and continued feedback to junior residents about their charting quality. ${ }^{23}$ O'Brien found that a quality assurance review was strengthened by relevant notices and reminders which were displayed around the clinic. ${ }^{26}$ Nallasivan emphasised the role of physician engagement and awareness about patient safety risk and the financial impact of miscoding to enhance physician chart documentation behaviour. ${ }^{27}$

Farzandipour et al. indicated that the compilation of high quality medical records relies not only on changing physician's behaviour, it also requires the support of medical record committees. ${ }^{28}$ Karami et al. found that physicians and administrators consider a combination of methods to be more effective than a single intervention for improving medical records documentation. ${ }^{29}$

\section{Shared responsibility}

These results and our observations strengthen the theory that high-quality medical record documentation is best achieved when it is a shared responsibility between organisational, clinical and administrative stakeholders. ${ }^{24-32}$ Healthcare practitioners are simply the most visible in the documentation process. High-quality medical records are more likely when healthcare practitioners, health information management 
TABLE I Scores for diagnostic accuracy by residents pre- and post- intervention

\begin{tabular}{|c|c|c|c|c|}
\hline Subspecialty & Criteria & $\begin{array}{l}\text { Pre- } \\
\text { intervention }\end{array}$ & $\begin{array}{l}\text { Post- } \\
\text { intervention }\end{array}$ & \\
\hline & & Mean/SD & Mean/SD & $p$ value \\
\hline \multirow[t]{2}{*}{ Internal } & Main condition & $3.25 \pm 1.9$ & $2.88 \pm 1.9$ & 0.285 \\
\hline & Other condition & $3.62 \pm 1.9$ & $3.25 \pm 1.7$ & 0.584 \\
\hline \multirow[t]{4}{*}{ Obstetric } & Type of delivery & $5 \pm 0 / 00$ & $5 \pm 0 / 00$ & - \\
\hline & Place of delivery & $5 \pm 0 / 00$ & $5 \pm 0 / 00$ & - \\
\hline & Outcome of delivery & $5 \pm 0 / 00$ & $5 \pm 0 / 00$ & - \\
\hline & Complication of delivery & $5 \pm 0 / 00$ & $5 \pm 0 / 00$ & - \\
\hline \multirow[t]{4}{*}{ Cancer surgery } & Location & $4.13 \pm 0.99$ & $4.63 \pm 1 / 0$ & 0.43 \\
\hline & Histology & $0.13 \pm 0.34$ & 0 & 0.351 \\
\hline & Behaviour & $I \pm 0.92$ & $1.25 \pm 0.8$ & 0.649 \\
\hline & Primary/secondary & $1.13 \pm 0.99$ & $1.38 \pm 0.7$ & 0.626 \\
\hline \multicolumn{2}{|l|}{ Total } & $8.74 \pm 1.31$ & $8.74 \pm 1.3$ & 0.886 \\
\hline
\end{tabular}

professionals and organisation/administrators work together, acting as a 'documentation triangle'. Intervention by one of these groups alone (physicians) may not ensure performance improvement. The role of health information management (HIM) professionals in particular should be highlighted. They serve as the custodians of medical records in healthcare environments. ${ }^{33}$

We therefore believe that one of the most effective ways of improving medical record documentation is to introduce a number of strategies (such as educational interventions, poster campaigns and information sessions), reinforced with the support of committees, strong leadership and regular feedback, all underpinned by the collective involvement of physicians, health information management professionals and administrators. Studies should be undertaken in order to determine the most effective combination of approaches. ${ }^{24,29-32}$

Based on our results we suggest the following factors as contributors:

- Administrative/management mechanisms for encouraging correct medical record documentation: introduction of formal incentive programmes e.g.pay-for-performance (P4P), pay-for-quality improvement (P4QI), and less formal rewards i.e. gift, certificate of recognition). Other more punitive policies for incomplete records could include withholding resident pay cheques or not allowing them to graduate. Fines and suspension of privileges could also be implemented. ${ }^{30}$

- Health information management professionals could introduce formal record completion policies; streamline medical record completion guidelines; complete audits of records for deficiencies and advise individual residents; organise an ongoing records review and feedback process; redesign forms to ensure that they are user-friendly; educate practitioners about clinical documentation practices; monitor and report improvement efforts. ${ }^{30}$

- Healthcare practioners could contribute to the creation of formal record completion policies; help to redesign forms so that they are practical and easy to use; educate more junior colleagues about clinical documentation practices; levy fines and determine incentive and puntive programmes. ${ }^{30}$

\section{Study limitations}

Our study was limited in several ways. First, there was minimal analysis of how the education intervention was implemented. We did not distribute any information handouts or pamphlets for future follow up but the research team provided a book written by them as a reference for further reading about medical record 
documentation. We didn't create posters or laminated pocket cards as visual aids and reminders of best practice. There were no external forces e.g. positioning the lecture as part of a quality assurance review as O'Brien did, or for continuous quality improvement as Tinsley did. The intervention was also not reinforced by support or feedback from senior colleagues. The number of charts that were reviewed per physician was probably not sufficient or truly representative of performance. Assessment of the intervention was limited to one month following baseline data collection, while previous studies performed chart reviews again six months later to determine if the physicians' behaviour had changed. The small number of residents sampled in the study might have biased the results and not given a true representation of the likelihood of residents changing their behavior (although other studies have also been conducted on limited numbers of subjects). We believe our findings were still valuable and are supported by current research findings and related literature.

\section{Impact in our hospital}

This small study was an initial phase of a large campaign at our university to improve chart documentation and completion. Further work is looking at the impact of having a medical secretary (with an associate degree in medical records) available to assist physicians in documenting their medical diagnoses. The ICD was placed at the nursing station to provide specific diagnostic information and codes. Medical record coders also provide appropriate feedback to physicians. A medical record committee has also been formed to investigate future initiatives, including the introduction of punitive actions. A complete guideline for recording diagnostic information and coding is also being prepared, based on the ICD, and will be distributed among residents in related subspecialties.

\section{CONCLUSION}

Physicians act as the author of individual patient medical records and define the majority of patient specific information. It is essential that all patient medical records are complete and accurate. They are relevant to the care of each individual patient but the data are also aggregated for public health use in preventing disease and promoting health. We would argue that the most effective ways of encouraging residents to learn, understand and implement best practice in medical record documentation is to develop systems and processes that are shared across the hospital between practitioners, administrators and information management specialists. These processes could include new forms for easy completion, open communication with senior colleagues and education campaigns. Compliance could be rewarded while incomplete records could result in punitive measures. The effectiveness of such processes must be quantified by structured research and not presumed to be successful.

9 Reid B.The impact of different coding systems on DRG assignment and data. Health Policy 1991; I7: 1 33-49.http://dx.doi.org//0.1016/01688510(9I)90050-8

10 Lemen PM. Development and assessment of a web-based evaluation and management coding curriculum for residents. Am J Obstet Gynecol 2005; 193:1785-9. http://dx.doi.org/10.1016/j. ajog.2005.08.005

II Wilchesky M,Tamblyn RM, Huang A.Validation of diagnostic codes within medical services claims. J Clin Epidemiol 2004; 57:|31-4I. http://dx.doi.org/I0.10I6/S0895-4356(03)00246-4

12 Farzandipour M, Sheikhtaheri A, Sadoughi F. Effective factors on accuracy of principal diagnosis coding based on International Classification of Diseases, the 10th revision (ICD-10). International Journal of Information Management 2010; 30: 78-84. http://dx.doi. org/I0.1016/j.ijinfomgt.2009.07.002

13 Farzandipour M, Sheikhtaheri A. Evaluation of factors influencing accuracy of principal procedure coding based on ICD-9-CM: an Iranian study. Perspect Health Inf Manag 2009 7; 6:5.

14 De Coster C, Quan H, Finlayson A et al. Identifying priorities in methodological research using ICD-9-CM and ICD-I0 administrative data: report from an international consortium. BMC Health Serv Res 2006; 6:77. http://dx.doi.org// 0. I I86//472-6963-6-77

I5 Langley J. Description and classification of childhood burns. Burns Incl Therm Inj 1984; 10:231-5. http://dx.doi.org/10.1016/03054I79(84)9000I-9

16 Farzandi pour M, Rabiee R, Ayatolahi $\mathrm{H}$ et al. Documention principles for medical record. Kashan: Morsal; 2005.

17 Shetty V, Murphy DA, Zigler C et al. Accuracy of data collected by surgical residents. J Oral Maxillofac Surg 2008; 66:1335-42. http:// dx.doi.org//0.1016/j.joms.2008.0I.065 
18 Bowman SM, Aitken ME. Assessing external cause of injury coding accuracy for transport injury hospitalizations. Perspect Health Inf Manag 201 I; 8: Ic.

19 As-Sanie S, Zolnoun D, Wechter ME et al. Teaching residents coding and documentation: effectiveness of a problem-oriented approach. Am J Obstet Gynecol 2005; 193:1790-3. http://dx.doi. org/I0.10I6/j.ajog.2005.08.004

20 Davis N, LaCour M. Introduction to health information technology. Philadelphia:WB Saunders and Company; 2002. p. 214.

21 Esmaeili MR, Abazari H, Mohammadi Kenari H. [Comparison of medical students' and pediatric residents' practices in medical records at Amirkola Children's Hospital]. Journal of Babol University of Medical Sciences 2010; 12: 106-II.

22 Seyf Rabiei MA, Sedighi I, Mazdeh MD et al. [Study of hospital records registration in teaching hospitals of Hamadan University of Medical Sciences in 2009]. Scientific Journal of Hamadan University of Medical Sciences and Health Services 2009; 16:45-9. [Persian].

23 Tinsley JA. An educational intervention to improve residents' inpatient charting. Acad Psychiatry 2004; 28:136-9. http://dx.doi. org/I0.1 I76/appi.ap.28.2.136

24 Socolar RR, Raines B, Chen-Mok M et al. Intervention to improve physician documentation and knowledge of child sexual abuse: a randomized, controlled trial. Pediatrics 1998; 101:817-24. http:// dx.doi.org/10.1542/peds.101.5.817

25 Greco PJ, Eisenberg JM. Changing physicians' practices. New EnglJ Med 1993; 329:127I-3. http://dx.doi.org/I0.1056/NEJMI993/02/32917/4

26 O'Brien KE, Chandramohan V, Nelson DA et al. Effect of a physician-directed educational campaign on performance of proper diabetic foot exams in an outpatient setting.J Gen Intern Med 2003; 18:258-65. http://dx.doi.org/I0.1046/j.1525-1497.2003.10662.x
27 Nallasivan S, Gillott T, Kamath S et al. Physician involvement enhances coding accuracy to ensure national standards: an initiative to improve awareness among new junior trainees. J $R$ Coll Physicians Edinb 201I; 4I:106-8. http://dx.doi.org//0.4997/ IRCPE.20II.220

28 Farzandipour M, Asef Zadeh S. [Evaluation of recording diagnosis principals in Kashan University's Hospitals]. Fayz 2004; 8:36-44. [Persian].

29 Karami M, Shokrizadeh Arani L. Related factors in medical records documentation quality and presenting solutions from managers' and physicians' viewpoints occupied in hospitals affiliated to Kashan University of Medical Sciences. Iranian Journal of Medical Education 2010; 9: 356-64.

30 Fletcher DM. Practice brief:best practices in medical record documentation and completion [Internet]. Chicago:AHIMA; 1999 [cited 2012 Feb 4]. Available from:http://www.ahima.org/Default.aspx

3I Davis DA, Thomson MA, Oxman AD et al. Changing physician performance. A systematic review of the effect of continuing medical education strategies. JAMA 1995; 274:700-5.http://dx.doi. org/ I0.100I/jama.1995.03530090032018

32 Smith WR.Evidence for the effectiveness of techniques to change physician behavior. Chest 2000;118:8S-17S. http://dx.doi. org/l0.1378/chest.1 I8.2_suppl.8S

33 Brodnik MS, Houser SH. Redefining the health information management scholar role. Perspect Health Inf Manag 2009; 16: le. 